\title{
Explanation of industrial analyses
}

\section{Input-Output Supply and Use tables}

The annual estimates prepared for the Blue Book incorporate the results of annual inquiries which become available in the first part of the year, although estimates for the latest year are still based largely on quarterly information. As new data are collected it is likely that revisions will be necessary. The process of reassessing these estimates involves the preparation of Supply and Use tables. This approach amalgamates all the available information on inputs, outputs, gross value added, income and expenditure. Similarly the production of the consolidated sector and financial accounts requires the preparation of 'top-to-bottom' sector and sub-sector accounts to identify discrepancies in the estimates relating to each sector. The thorough and detailed nature of this estimation process require a large amount of time and resource and so estimates for earlier years are not normally revisited unless there are strong reasons for doing so.

\section{GDP and the balancing of the annual accounts}

As discussed in Part 1, there are three different approaches to the estimation of current price GDP in the UK: the income approach, the expenditure approach and the production approach. In theory the three different approaches should produce the same result. However, the different approaches are based on different surveys and administrative data sources and each produces estimates which, like all statistical estimates, are subject to errors and omissions. A definitive GDP estimate can only emerge after a process of balancing and adjustment. ONS believes that the most reliable 'definitive' estimate of the current price level of GDP is that derived using the annual Supply and Use tables framework. Thus, for the years when Supply and Use tables are available, GDP is set at the level derived from that year's balance. For periods subsequent to the latest Supply and Use tables, the level of GDP is carried forward using movements in income, expenditure and production totals.

\section{The Supply and Use framework}

The accounting framework shown in Figure 1 in the Introduction is mainly concerned with the composition and value of goods and services entering into final demand (for example, purchases by consumers) and the outputs and incomes generated in the production process. It does not display the inter-industry transactions which link these activities.

The UK Supply and Use tables, however, do include these intermediate transactions which form inputs into these processes, thus providing an extra dimension. The analyses are constructed to show a balanced and complete picture of the flows of products in the economy and illustrate the relationships between producers and consumers of goods and services. On an annual basis, Supply and Use tables are used to achieve consistency in the economic accounts' aggregates by linking the components of value added, inputs, outputs and final demand. As the income, production and expenditure measures of GDP can all be calculated from the Supply and Use tables, a single estimate of GDP can be derived by balancing the supply and demand for goods and services and reconciling them with the corresponding value added estimates. For the years 1989 to 2008, the balancing process has been used to set the level of current price GDP and has disposed of the need for statistical discrepancies in the form of a GDP expenditure adjustment and a GDP income adjustment.

\section{Industrial analyses}

The process, which produces Supply and Use tables annually, has been speeded up considerably over the last few years and can now produce the first balance for a year around 18 months after the end of that year. These full Supply and Use tables, consistent with the National Accounts Blue Book, are published as a separate web-only publication at the same time as the Blue Book. The latest annual Supply and Use tables ${ }^{1}$ cover the periods 2006 to 2008, with summary information provided in the Blue Book itself.

Some background on the structure of the Supply and Use tables

The Supply and Use tables are based on a framework which incorporates estimates of industry inputs, outputs and value added. The tables consist of two matrices: the Supply table 
and the Use table, each of which breaks down and balances 108 different industries and 123 products at purchasers' prices. The following paragraphs summarise the methodology. For more detail see Akers and Clifton-Fearnside (2008). ${ }^{2}$

\section{Supply table}

At a very aggregate level the Supply table can be represented as follows:

\begin{tabular}{|l|l|l|l|l|}
\cline { 2 - 4 } \multicolumn{1}{c|}{} & $\begin{array}{l}\text { Output by } \\
\text { industry }\end{array}$ & $\begin{array}{l}\text { Imports of } \\
\text { goods and } \\
\text { services }\end{array}$ & $\begin{array}{l}\text { Dis- } \\
\text { tributors' } \\
\text { trading } \\
\text { margins }\end{array}$ & $\begin{array}{l}\text { Taxes } \\
\text { less } \\
\text { subsidies } \\
\text { on } \\
\text { products }\end{array}$ \\
\hline $\begin{array}{l}\text { Output } \\
\text { by } \\
\text { product }\end{array}$ & & & & \\
\hline
\end{tabular}

The main part of the Supply table shows estimates of domestic industries' output (total sales adjusted for changes in inventories of work in progress and finished goods) compiled at basic prices. Basic prices value the goods leaving the factory gate but exclude any taxes on products and include any subsidies on products. However, for the balancing process, the estimates of supply of products are required at purchasers' prices, that is, those actually paid by the purchasers to take delivery of the goods, excluding any deductible VAT. To convert the estimates of domestic output valued at basic prices to the total supply of products valued at purchasers' prices requires the addition of:

- the value of imports of goods and services

- distributors' trading margins

- taxes on products (for example, VAT, excise duties, air passenger tax and insurance premium tax)

less

- subsidies on products (for example, agricultural and transport subsidies)

\section{Use table}

The Use table reveals the input structure of each industry in terms of combined domestic and imported goods and services.

Industry consumption/final demand table
It also shows the product composition of final demand and, for each industry, the intermediate purchases adjusted for changes in inventories of materials and fuels. Consumption of products is represented in the rows of the balance while purchases by industries, and final demands, are represented in the columns. At a very aggregate level the Use table can be considered in three parts as shown below.

The body of the matrix, which represents consumption of products, is at purchasers' prices and so already includes the product-specific taxes and subsidies separately added in the Supply table.

The Supply-Use balance is effectively achieved when:

For industries:

Inputs (from the Use table)

equals

Outputs (from the Supply table)

For products:

Supply (from the Supply table)

equals

Demand (from the Use table)

That is, when the data from the income, expenditure and production approaches used to fill the matrices all produce the same estimate of current price GDP at market prices. GDP at current market prices can be derived from the balances by taking the estimate of total gross value added at basic prices (from the Use table) and adding taxes on products and deducting subsidies on products (from the Supply table).

\section{The balancing process}

The Supply and Use tables produced this year use the same methods first employed in the 2008 edition of the Blue Book (see Akers and Clifton-Fearnside (2008)2). A detailed description of the methods and process used for the 2006, and earlier, editions of the Blue Book can be found in Mahajan $(2006)^{3}$.

The current approach utilises new computing systems resulting from the ONS programme for the modernisation of its statistical systems and processes. The process of balancing

\begin{tabular}{|l|l|l|}
\cline { 2 - 3 } \multicolumn{1}{c|}{} & Industry consumption & Final demand \\
\hline Products consumed & $\begin{array}{l}\text { Shows consumption by each industry to } \\
\text { produce their own output } \\
\text { (that is, intermediate consumption) }\end{array}$ & $\begin{array}{l}\text { Shows final demand categories (for example, } \\
\text { households' expenditure) and the values of } \\
\text { products going to these categories }\end{array}$ \\
\hline Primary inputs & $\begin{array}{l}\text { Shows the gross value added components } \\
\text { of each industry, taxes less subsidies on } \\
\text { production other than product specific } \\
\text { taxes and subsidies, compensation of } \\
\text { employees and gross operating surplus }\end{array}$ & \\
\hline
\end{tabular}


includes the compilers of the basic data that form the input to the process. These individuals bring with them an understanding of the data that is being used to populate the Supply and Use framework. If we consider the Supply and Use framework as a column (industry) and row (product) matrix the process of balancing consists of a series of row and column confrontations of the data.

The first interrogation of data takes place before the Supply and Use framework is populated. It consists of an examination of the columns in the framework being reviewed for plausibility independently of each other. For example, estimates of household consumption expenditure, by product, are produced and analysed to ensure the overall picture of household spending and its breakdown by product presents a credible story in their own right. Similarly, for those components with an industry dimension, such as output, the initial stage scrutinises these data to ensure the story for industries look plausible. This first stage is carried out by the compilers of the original data.

The second stage is a confrontation within the framework of the rows (products) in the Supply and Use framework. The accounting relationship that supply is equal to demand is tested for each product. This process identifies areas of inconsistency between the various sources which can then be investigated. Data within the row are then subsequently adjusted to achieve a balance. This adjustment process reviews the quality of the data used to populate the individual cells within a row and makes use of this information to adjust the original data.

The third stage of the balancing process is to confront the columns. Unlike the interrogation within the column, carried out during the first stage, this time it is carried out in the context of the Supply and Use framework. While the second stage of balancing results in a balance of the rows, it does not result in satisfying the accounting relationship for the columns. This needs to ensure that for each industry the inputs to the process of production equal its outputs. This third stage of balancing has the objective of confirming that this column identity is satisfied.

Once stage three is complete there is a strong probability that the row identities balanced during stage two of the process will have subsequently been broken. The process of repeating stages two and three continues until both the row and column identities are satisfied. This iterative process of row and column balancing effectively homes in on a position of balance by way of narrowing the degree of imbalance remaining in the Supply and Use framework after each balancing cycle. While the description above may seem to indicate a fairly mechanistic balancing approach, a significant amount of knowledge of the methods and quality of the basic data are used as part of the process. Alongside this, the evolving balance is reviewed at each stage to see how the economic story is developing and make sure that story is credible.

\section{Current price analysis (Tables 2.1, 2.2 and 2.3)}

The analyses of gross value added and other variables by industry shown in Part 2 reflect estimates based on the Standard Industrial Classification (SIC(2003)). Tables 2.1, 2.2 and 2.3 are based on current price data reconciled through the Input-Output (I-O) process for 1992 to 2008.

Estimates of total output and gross value added are valued at basic prices, the method recommended by ESA95 (the European System of National and Regional Accounts, 1995). Thus the only taxes included in the price will be taxes paid as part of the production process (such as business rates and vehicle excise duty), and not any taxes specifically levied on the production of a unit of output (such as VAT). Any subsidies on products received will also be included in the valuation of output.

\section{Chained volume indices $(2006=100)$ analyses (Table 2.4)}

Table 2.4 shows chained volume estimates of gross value added at basic prices by industry. The source data for these estimates can be found in the $\operatorname{GDP}(\mathrm{O})$ source data guide on the ONS website:

\section{www.ons.gov.uk/about-statistics/user-guidance/ ios-methodology/source-data/index.html}

A more detailed explanation is in Concepts, Sources and Methods. ${ }^{4}$

The output approach provides the lead indicator of economic change in the short-term. However in the longer-term, it is required to follow the annual path indicated by the expenditure measure of real GDP (usually within 0.2 per cent of the average annual gross value added growth). To achieve this, balancing adjustments are sometimes applied to the output based gross value added estimates.

An examination of the chained volume gross value added and expenditure measures of GDP shows what are considered to be excessive differences in growth for a number of recent years.

The output-based estimate grew less quickly than the expenditure measure in 2006 but more quickly in 2007 and 2008.

The largest difference in growth between the output and expenditure GVA measure was 0.6 per cent, which occurred in 2008. 
To reduce these discrepancies, a number of balancing adjustments have been made to the chained volume gross value added annual growth rates.

Assigning adjustments: improvements for the 2010 Blue Book

For this year's Blue Book, balancing adjustments have been applied on the same basis as for the 2009 Blue Book. For technical and other reasons, the adjustments are not at present made to the non-service industries for any years.

\section{Applying the adjustments}

ONS has developed an automatic function for assigning the annual adjustments to gross value added. This is designed to be as faithful as possible to the quarterly paths whilst adjusting the overall annual growth rate of a group of series. Details of the new adjustments are given below. Using the automatic function produces some differences in the adjustments within the groupings shown.

\section{For 2006:}

An upwards adjustment of 0.1 per cent has been applied to:

- distribution, hotels and restaurants; (sections $\mathrm{G}$ and $\mathrm{H}$ )

An upwards adjustment of 0.3 per cent has been applied to:

- transport, storage and communication (section I)

An upwards adjustment of 0.3 per cent has been applied to:

- Business Services and Finance (section J and K)

An upwards adjustment of 0.3 per cent has been applied to:

- government and other services (sections $L$ to $P$ )

The total effect of these adjustments is to increase the 2006 growth rate by 0.2 per cent.

\section{For 2007:}

A downwards adjustment of -0.1 per cent has been applied to:

- distribution, hotels and restaurants; (sections $\mathrm{G}$ and $\mathrm{H}$ )

A downwards adjustment of -0.2 per cent has been applied to:

- transport, storage and communication (section I)

A downwards adjustment of -0.2 per cent has been applied to:

- Business Services and Finance (section J and K)

A downwards adjustment of -0.2 per cent has been applied to:

- government and other services (sections $L$ to $P$ )

The total effect of these adjustments is to decrease the 2007 growth rate by 0.1 per cent.

\section{For 2008:}

A downwards adjustment of -0.3 per cent has been applied to:

distribution, hotels and restaurants; (sections $\mathrm{G}$ and $\mathrm{H}$ )

A downwards adjustment of -0.9 per cent has been applied to:

- transport, storage and communication (section I)

A downwards adjustment of -0.9 per cent has been applied to:

Business Services and Finance (section J and K)

A downwards adjustment of -0.9 per cent has been applied to:

- government and other services (sections L to P)

The total effect of these adjustments is to decrease the 2008 growth rate by -0.6 per cent.

\section{Workforce jobs by industry (Table 2.5)}

Workforce Jobs (WFJ) is the preferred measure of the change in employment by industry. The number of jobs is not the same as the number of people employed. This is because a person can have more than one job.

Table 2.5 breaks down WFJ into six broad industry groupings on SIC 2003. WFJ is also now available on SIC 2007, but SIC 2003 has been used for this table to be consistent with other Blue Book tables.

Employee jobs, the main component of WFJ are obtained mainly from surveys of businesses selected from the InterDepartmental Business Register (IDBR). This is the same register used for all other business surveys collecting economic data.

Self-employment jobs come from the Labour Force Survey (LFS). This is a household survey which codes respondents according to their own view of the industry in which they work and so the industry breakdown is less reliable than the business surveys.

WFJ also includes Her Majesty's Forces (contained within industry section $\mathrm{L}$ ) and government supported trainees from administrative sources (split by industry using the LFS).

\section{References:}

1 Office for National Statistics (2010) Supply-Use Tables 2010: www.statistics.gov.uk/about/methodology_by_theme/ inputoutput/latestdata.asp

2 Akers R and Clifton-Fearnside A (2008): www.statistics.gov.uk/about/methodology_by_theme/ inputoutput/articles_and_analyses.asp

3 Mahajan S (2006) Compilation and Use of Input-Output Supply and Use Tables in the UK National Accounts Economic Trends No. 634. 
4 Office for National Statistics (1998) National Accounts Concepts, Sources and Methods, 1998 edition.

The Stationery Office: London. 


\begin{tabular}{|c|c|c|c|c|c|c|}
\hline \multirow{3}{*}{2006} & \multicolumn{6}{|c|}{ SUPPLY OF PRODUCTS } \\
\hline & \multirow{2}{*}{$\begin{array}{r}\text { Domestic } \\
\text { output of } \\
\text { products } \\
\text { at } \\
\text { basic prices }\end{array}$} & \multicolumn{2}{|c|}{ Imports } & \multirow{2}{*}{$\begin{array}{r}\text { Distributors' } \\
\text { trading } \\
\text { margins }\end{array}$} & \multirow{2}{*}{$\begin{array}{r}\text { Taxes } \\
\text { less } \\
\text { subsidies } \\
\text { on } \\
\text { products }\end{array}$} & \multirow{2}{*}{$\begin{array}{r}\text { Total } \\
\text { supply of } \\
\text { products at } \\
\text { purchasers } \\
\text { prices }\end{array}$} \\
\hline & & Goods & Services & & & \\
\hline \multicolumn{7}{|l|}{ PRODUCTS ${ }^{1}$} \\
\hline Agriculture, forestry \& fishing $[1-3]$ & 19043 & 7173 & 421 & 4106 & 466 & 31209 \\
\hline Mining \& quarrying [4-7] & 42172 & 24218 & 552 & 1863 & 461 & 69266 \\
\hline Manufacturing [8-84] & 409220 & 284922 & 16270 & 225977 & 83180 & 1019569 \\
\hline Electricity, gas \& water supply [85-87] & 68361 & 399 & 64 & - & 2611 & 71435 \\
\hline Construction [88] & 194310 & - & 692 & - & 15194 & 210196 \\
\hline Distribution \& hotels [89-92] & 347901 & - & 14422 & -231946 & 13518 & 143895 \\
\hline Transport \& communication [93-99] & 181363 & - & 20453 & - & 1760 & 203576 \\
\hline Finance \& business services $[100-114]$ & 645982 & 298 & 39738 & - & 17793 & 703811 \\
\hline Public administration \& defence [115] & 129801 & - & 61 & - & & 129862 \\
\hline Education, health \& social work [116-118] & 259680 & - & 1987 & - & 2267 & 263934 \\
\hline Other services [119-123] & 100284 & 2935 & 4958 & - & 7409 & 115586 \\
\hline Total & 2398117 & 319945 & 99618 & - & 144659 & 2962339 \\
\hline \multicolumn{7}{|l|}{ of which: } \\
\hline Market output & 1977807 & & & & & \\
\hline Output for own final use & 102751 & & & & & \\
\hline Other non-market output & 317559 & & & & & \\
\hline
\end{tabular}

\section{Use Table at Purchasers' prices}

\begin{tabular}{|c|c|c|c|c|c|c|c|c|c|c|c|}
\hline \multirow[b]{2}{*}{2006} & \multicolumn{11}{|c|}{ INTERMEDIATE CONSUMPTION BY INDUSTRY GROUP ${ }^{12}$} \\
\hline & Agriculture & $\begin{array}{c}\text { Mining \& } \\
\text { quarrying }\end{array}$ & $\begin{array}{r}\text { Manufac- } \\
\text { turing }\end{array}$ & $\begin{array}{r}4 \\
\text { Electricity, } \\
\text { gas \& } \\
\text { water } \\
\text { supply }\end{array}$ & $\begin{array}{r}5 \\
\begin{array}{r}\text { Construc- } \\
\text { tion }\end{array}\end{array}$ & $\begin{array}{r}6 \\
\text { Distribution } \\
\& \\
\text { hotels }\end{array}$ & $\begin{array}{r}7 \\
\text { Transport } \\
\& \\
\text { communi- } \\
\text { cation }\end{array}$ & $\begin{array}{r}8 \\
\text { Finance } \\
\& \\
\text { business } \\
\text { services }\end{array}$ & $\begin{array}{r}9 \\
\text { Public } \\
\text { adminis- } \\
\text { tration \& } \\
\text { defence }\end{array}$ & $\begin{array}{r}10 \\
\text { Education, } \\
\text { health \& } \\
\text { social work }\end{array}$ & $\begin{array}{r}\text { Other } \\
\text { services }\end{array}$ \\
\hline \multicolumn{12}{|l|}{ PRODUCTS ${ }^{1}$} \\
\hline Agriculture, forestry \& fishing [1-3] & 2173 & 1 & 10375 & 10 & 242 & 1802 & 45 & 13 & 2 & 261 & 40 \\
\hline Mining \& quarrying [4-7] & 9 & 3943 & 20684 & 21097 & 2977 & 241 & 102 & 17 & - & 9 & 60 \\
\hline Manufacturing [8-84] & 6265 & 2498 & 191746 & 4293 & 29368 & 48624 & 22989 & 14742 & 23761 & 31635 & 7490 \\
\hline Electricity, gas \& water supply [85-87] & 488 & 898 & 11647 & 20095 & 309 & 2370 & 1056 & 1775 & 1353 & 2257 & 717 \\
\hline Construction [88] & 267 & 924 & 1445 & 925 & 56111 & 1786 & 2209 & 12767 & 5070 & 1074 & 663 \\
\hline Distribution \& hotels [89-92] & 699 & 109 & 1434 & 264 & 1868 & 7462 & 3463 & 6592 & 1856 & 2479 & 765 \\
\hline Transport \& communication [93-99] & 473 & 1347 & 14534 & 413 & 1392 & 32667 & 39201 & 27019 & 5282 & 5826 & 2846 \\
\hline Finance \& business services $[100-114]$ & 2246 & 2980 & 34816 & 3784 & 23892 & 60291 & 29636 & 162640 & 21546 & 23754 & 19209 \\
\hline Public administration \& defence [115] & 12 & 24 & 564 & 53 & 405 & 251 & 2016 & 7191 & 294 & 97 & 77 \\
\hline Education, health \& social work [116-118] & 192 & 37 & 1302 & 223 & 192 & 1049 & 1270 & 5642 & 4945 & 34164 & 1028 \\
\hline Other services [119-123] & 267 & 113 & 3376 & 191 & 177 & 2019 & 1959 & 4349 & 3468 & 3692 & 17269 \\
\hline Total consumption & 13091 & 12874 & 291923 & 51348 & 116933 & 158562 & 103946 & 242747 & 67577 & 105248 & 50164 \\
\hline Taxes less subsidies on production & -3039 & 276 & 2445 & 1149 & 668 & 8423 & 1179 & 1936 & - & 343 & 1139 \\
\hline Compensation of employees & 3757 & 3504 & 108032 & 5146 & 36392 & 109702 & 55362 & 166422 & 53573 & 133217 & 37901 \\
\hline Gross operating surplus & 7070 & 27972 & 40978 & 13984 & 37559 & 51835 & 27114 & 198557 & 8651 & 21331 & 21126 \\
\hline Gross value added at basic prices & 7788 & 31752 & 151455 & 20279 & 74619 & 169960 & 83655 & 366915 & 62224 & 154891 & 60166 \\
\hline Output at basic prices & 20879 & 44626 & 443378 & 71627 & 191552 & 328522 & 187601 & 609662 & 129801 & 260139 & 110330 \\
\hline
\end{tabular}


continued

\section{Gross value added at basic prices}

f billion

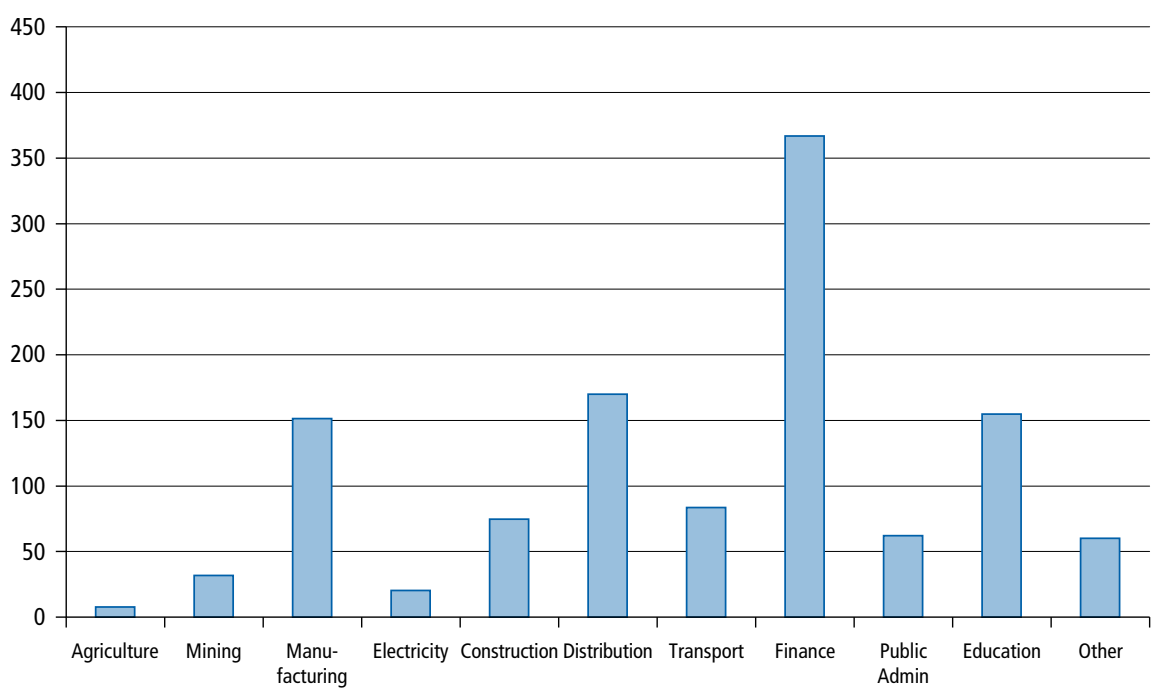

\section{Components of final demand}

Per cent

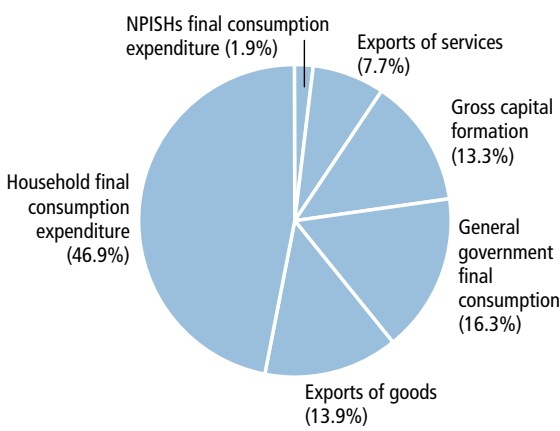

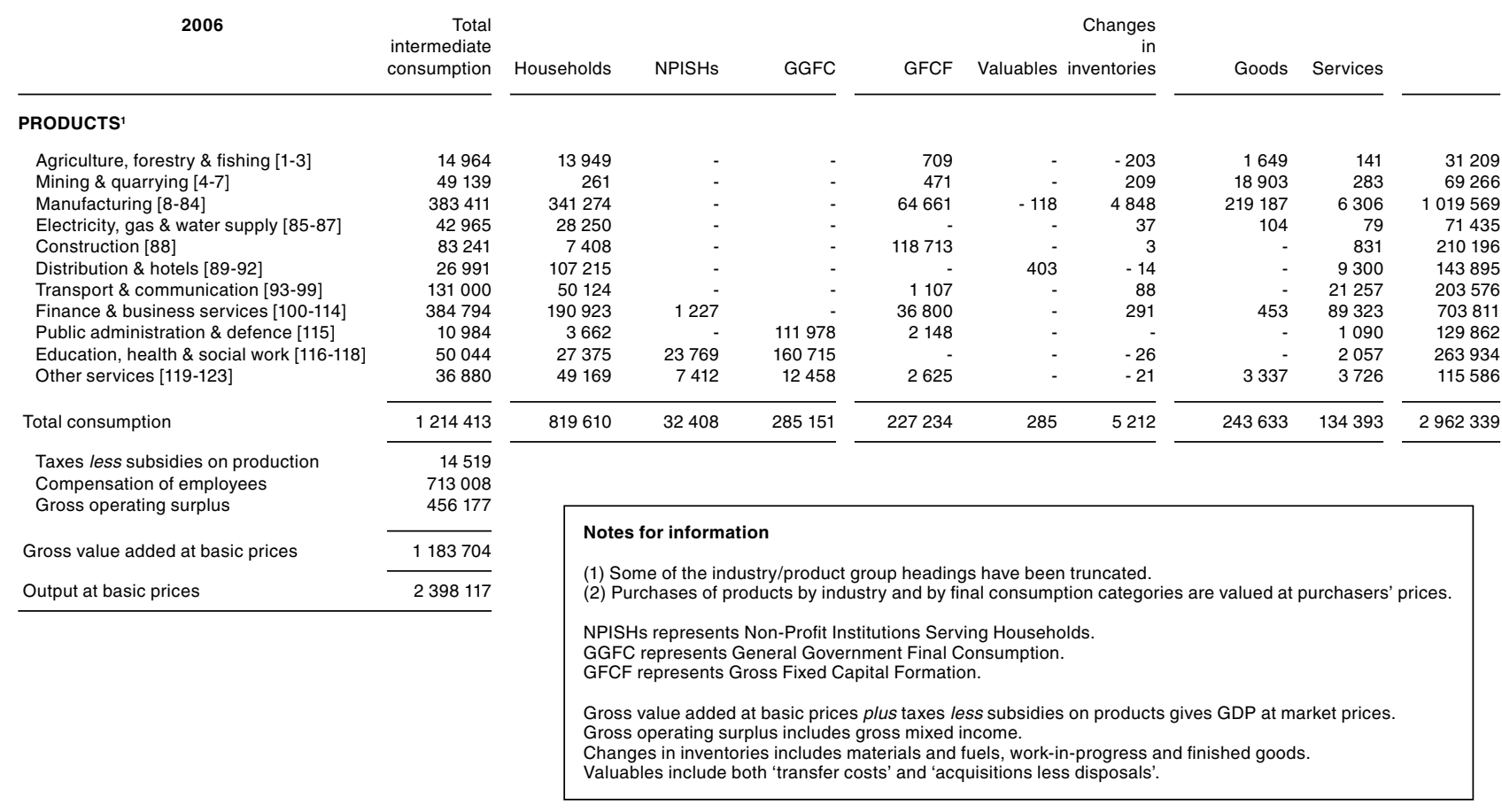




\begin{tabular}{|c|c|c|c|c|c|c|}
\hline \multirow{3}{*}{2007} & \multicolumn{6}{|c|}{ SUPPLY OF PRODUCTS } \\
\hline & \multirow{2}{*}{$\begin{array}{r}\text { Domestic } \\
\text { output of } \\
\text { products } \\
\text { at } \\
\text { basic prices }\end{array}$} & \multicolumn{2}{|c|}{ Imports } & \multirow{2}{*}{$\begin{array}{r}\text { Distributors' } \\
\text { trading } \\
\text { margins }\end{array}$} & \multirow{2}{*}{$\begin{array}{r}\text { Taxes } \\
\text { less } \\
\text { subsidies } \\
\text { on } \\
\text { products }\end{array}$} & \multirow{2}{*}{$\begin{array}{r}\text { Total } \\
\text { supply of } \\
\text { products at } \\
\text { purchasers } \\
\text { prices }\end{array}$} \\
\hline & & Goods & Services & & & \\
\hline \multicolumn{7}{|l|}{ PRODUCTS ${ }^{1}$} \\
\hline Agriculture, forestry \& fishing $[1-3]$ & 21219 & 7358 & 443 & 4624 & 390 & 34034 \\
\hline Mining \& quarrying [4-7] & 42263 & 23828 & 613 & 1913 & 478 & 69095 \\
\hline Manufacturing [8-84] & 421911 & 275177 & 16820 & 236824 & 85305 & 1036037 \\
\hline Electricity, gas \& water supply [85-87] & 72792 & 232 & 69 & - & 2698 & 75791 \\
\hline Construction [88] & 214502 & - & 875 & - & 16888 & 232265 \\
\hline Distribution \& hotels [89-92] & 364028 & - & 14954 & -243361 & 14024 & 149645 \\
\hline Transport \& communication [93-99] & 190233 & - & 20730 & - & 3360 & 214323 \\
\hline Finance \& business services [100-114] & 695395 & 228 & 44385 & - & 19956 & 759964 \\
\hline Public administration \& defence [115] & 132326 & - & 61 & - & & 132387 \\
\hline Education, health \& social work [116-118] & 274896 & - & 2093 & - & 2385 & 279374 \\
\hline Other services [119-123] & 104748 & 3789 & 5304 & - & 7657 & 121498 \\
\hline Total & 2534313 & 310612 & 106347 & - & 153141 & 3104413 \\
\hline \multicolumn{7}{|l|}{ of which: } \\
\hline Market output & 2094879 & & & & & \\
\hline Output for own final use & 109034 & & & & & \\
\hline Other non-market output & 330400 & & & & & \\
\hline
\end{tabular}

\section{Use Table at Purchasers' prices}

\begin{tabular}{|c|c|c|c|c|c|c|c|c|c|c|c|}
\hline \multirow[b]{2}{*}{2007} & \multicolumn{11}{|c|}{ INTERMEDIATE CONSUMPTION BY INDUSTRY GROUP ${ }^{12}$} \\
\hline & Agriculture & $\begin{array}{c}\text { Mining \& } \\
\text { quarrying }\end{array}$ & $\begin{array}{r}\text { Manufac- } \\
\text { turing }\end{array}$ & $\begin{array}{r}4 \\
\text { Electricity, } \\
\text { gas \& } \\
\text { water } \\
\text { supply }\end{array}$ & $\begin{array}{r}5 \\
\begin{array}{r}\text { Construc- } \\
\text { tion }\end{array}\end{array}$ & $\begin{array}{r}6 \\
\text { Distribution } \\
\& \\
\text { hotels }\end{array}$ & $\begin{array}{r}7 \\
\text { Transport } \\
\& \\
\text { communi- } \\
\text { cation }\end{array}$ & $\begin{array}{r}8 \\
\text { Finance } \\
\& \\
\text { business } \\
\text { services }\end{array}$ & $\begin{array}{r}9 \\
\text { Public } \\
\text { adminis- } \\
\text { tration \& } \\
\text { defence }\end{array}$ & $\begin{array}{r}10 \\
\text { Education, } \\
\text { health \& } \\
\text { social work }\end{array}$ & $\begin{array}{r}\text { Other } \\
\text { services }\end{array}$ \\
\hline \multicolumn{12}{|l|}{ PRODUCTS ${ }^{1}$} \\
\hline Agriculture, forestry \& fishing [1-3] & 2446 & 1 & 11420 & 10 & 272 & 1861 & 57 & 15 & - & 277 & 42 \\
\hline Mining \& quarrying [4-7] & 9 & 3873 & 20771 & 22493 & 2907 & 237 & 96 & 17 & - & 9 & 56 \\
\hline Manufacturing [8-84] & 7231 & 2824 & 198710 & 4211 & 33000 & 51442 & 24081 & 15650 & 23556 & 34311 & 7660 \\
\hline Electricity, gas \& water supply [85-87] & 524 & 964 & 11707 & 21996 & 348 & 2454 & 1079 & 1915 & 1395 & 2414 & 750 \\
\hline Construction [88] & 274 & 823 & 1075 & 653 & 62757 & 1841 & 2257 & 14206 & 5192 & 1153 & 708 \\
\hline Distribution \& hotels [89-92] & 724 & 144 & 1488 & 301 & 1976 & 7762 & 3660 & 6747 & 1915 & 2690 & 796 \\
\hline Transport \& communication [93-99] & 503 & 1379 & 14923 & 418 & 1567 & 34055 & 41067 & 28206 & 5368 & 6208 & 2899 \\
\hline Finance \& business services $[100-114]$ & 2458 & 3650 & 36042 & 3959 & 26761 & 63562 & 30966 & 172660 & 22824 & 25766 & 20009 \\
\hline Public administration \& defence [115] & 14 & 29 & 602 & 53 & 456 & 269 & 2158 & 8028 & 298 & 101 & 80 \\
\hline Education, health \& social work [116-118] & 204 & 52 & 1398 & 220 & 216 & 1108 & 1297 & 6091 & 5006 & 36308 & 1067 \\
\hline Other services [119-123] & 289 & 143 & 3509 & 180 & 197 & 2105 & 1978 & 4742 & 3464 & 3906 & 17558 \\
\hline Total consumption & 14676 & 13882 & 301645 & 54494 & 130457 & 166696 & 108696 & 258277 & 69018 & 113143 & 51625 \\
\hline Taxes less subsidies on production & -2755 & 210 & 2354 & 1132 & 792 & 8723 & 831 & 2366 & - & 340 & 1097 \\
\hline Compensation of employees & 4100 & 3736 & 108891 & 6032 & 41125 & 115171 & 58830 & 181073 & 54245 & 138945 & 40066 \\
\hline Gross operating surplus & 7283 & 26903 & 43481 & 14720 & 38758 & 53803 & 28619 & 217247 & 9063 & 22862 & 21661 \\
\hline Gross value added at basic prices & 8628 & 30849 & 154726 & 21884 & 80675 & 177697 & 88280 & 400686 & 63308 & 162147 & 62824 \\
\hline Output at basic prices & 23304 & 44731 & 456371 & 76378 & 211132 & 344393 & 196976 & 658963 & 132326 & 275290 & 114449 \\
\hline
\end{tabular}


continued

\section{Gross value added at basic prices}

f billion

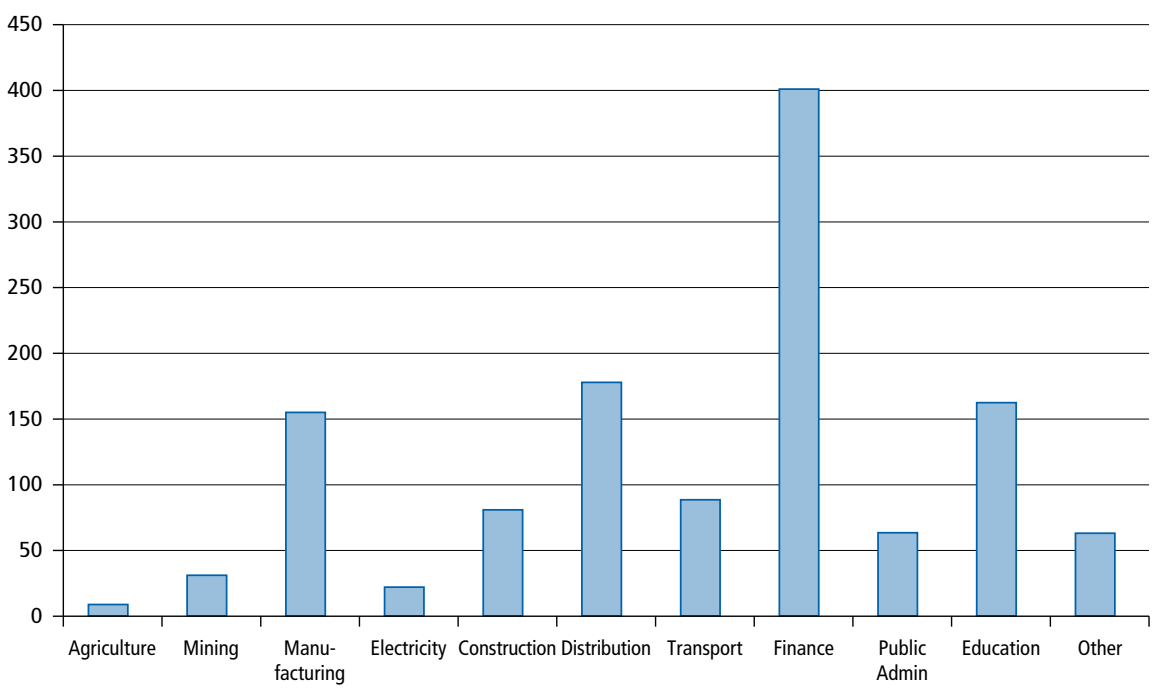

\section{Components of final demand}

Per cent

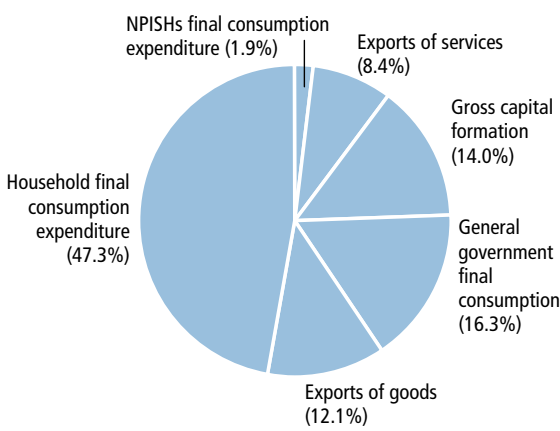

\section{FINAL CONSUMPTION EXPENDITURE ${ }^{2}$ GROSS CAPITAL FORMATION}

million

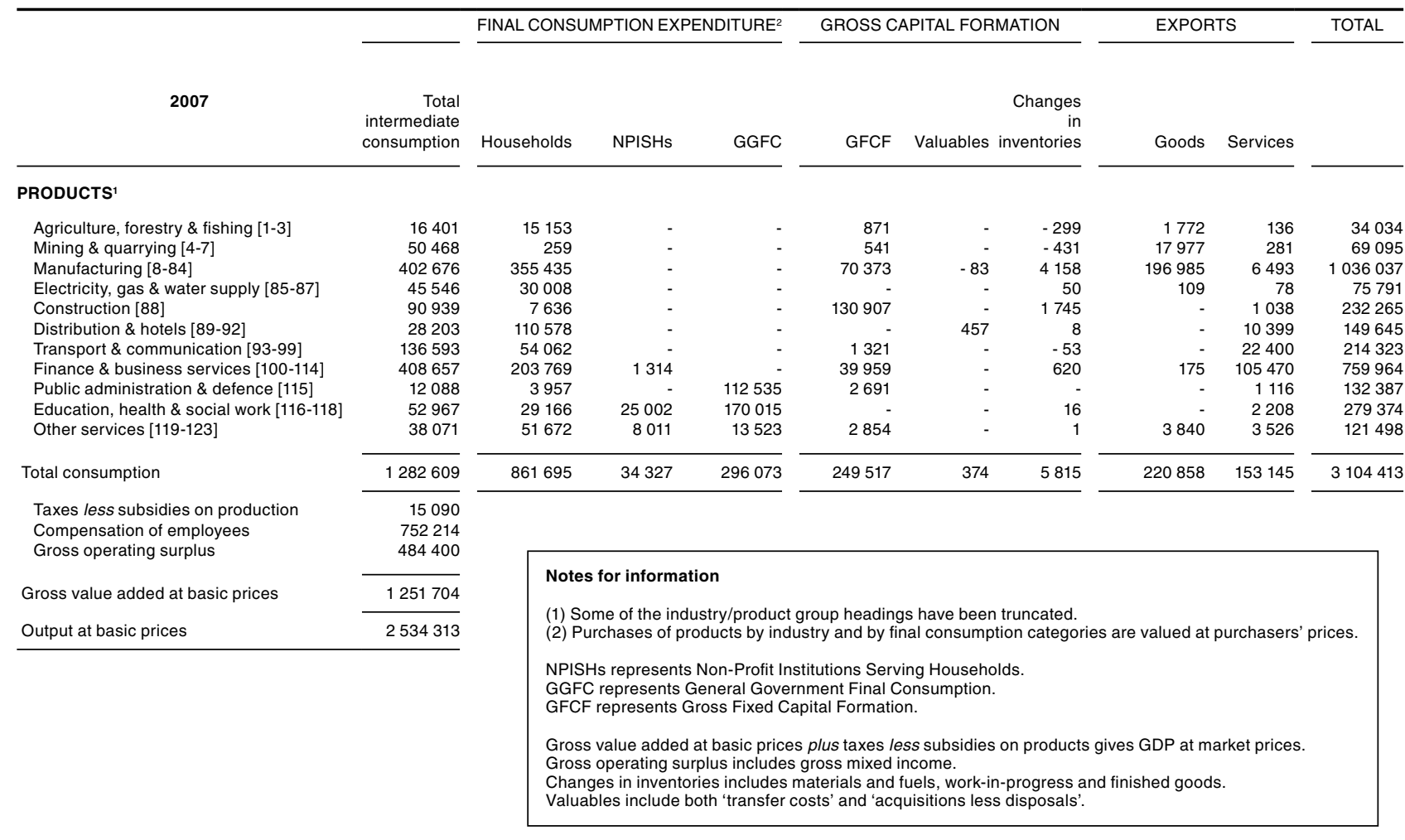




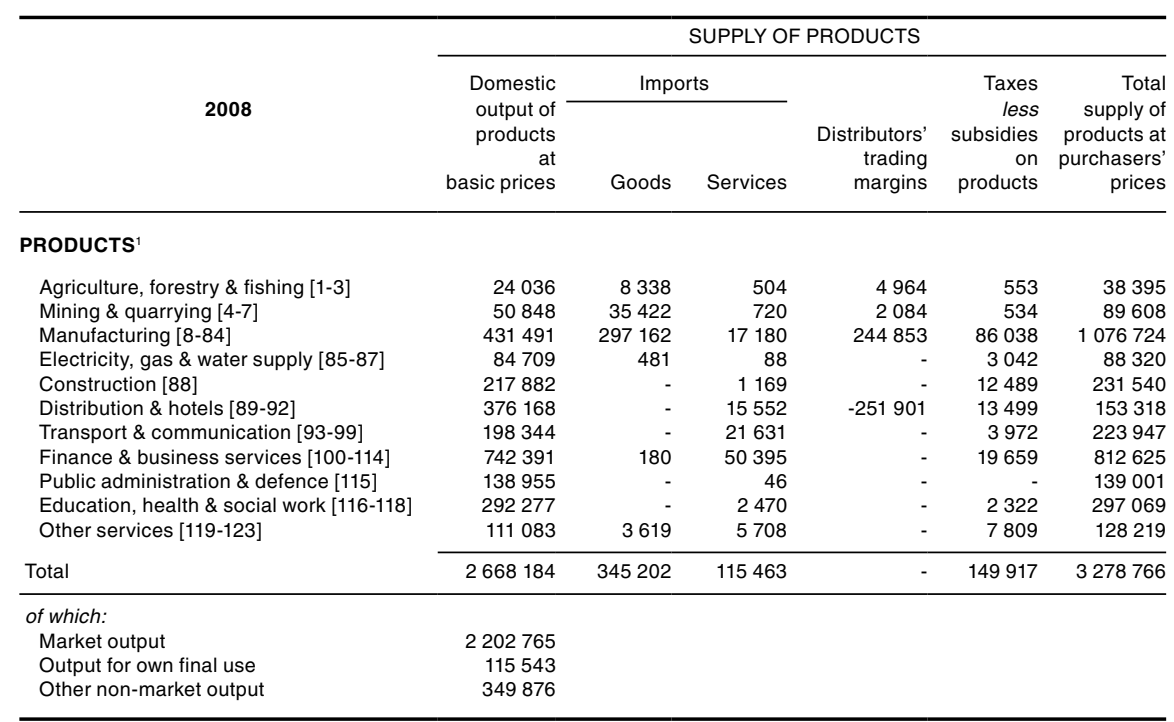

\section{Use Table at Purchasers' prices}

\begin{tabular}{|c|c|c|c|c|c|c|c|c|c|c|c|}
\hline \multirow[b]{2}{*}{2008} & \multicolumn{11}{|c|}{ INTERMEDIATE CONSUMPTION BY INDUSTRY GROUP ${ }^{12}$} \\
\hline & Agriculture & $\begin{array}{c}\text { Mining \& } \\
\text { quarrying }\end{array}$ & $\begin{array}{r}\text { Manufac- } \\
\text { turing }\end{array}$ & $\begin{array}{r}4 \\
\text { Electricity, } \\
\text { gas \& } \\
\text { water } \\
\text { supply }\end{array}$ & $\begin{array}{r}5 \\
\begin{array}{r}\text { Construc- } \\
\text { tion }\end{array}\end{array}$ & $\begin{array}{r}6 \\
\text { Distribution } \\
\& \\
\text { hotels }\end{array}$ & $\begin{array}{r}7 \\
\text { Transport } \\
\& \\
\text { communi- } \\
\text { cation }\end{array}$ & $\begin{array}{r}8 \\
\text { Finance } \\
\& \\
\text { business } \\
\text { services }\end{array}$ & $\begin{array}{r}9 \\
\text { Public } \\
\text { adminis- } \\
\text { tration \& } \\
\text { defence }\end{array}$ & $\begin{array}{r}10 \\
\text { Education, } \\
\text { health \& } \\
\text { social work }\end{array}$ & $\begin{array}{r}\text { Other } \\
\text { services }\end{array}$ \\
\hline \multicolumn{12}{|l|}{ PRODUCTS ${ }^{1}$} \\
\hline Agriculture, forestry \& fishing [1-3] & 2940 & 2 & 12343 & 10 & 300 & 2000 & 76 & 18 & - & 314 & 50 \\
\hline Mining \& quarrying [4-7] & 8 & 5135 & 27093 & 28873 & 2919 & 231 & 93 & 17 & - & 9 & 58 \\
\hline Manufacturing [8-84] & 8151 & 3156 & 201896 & 5038 & 33839 & 52652 & 25298 & 16992 & 24756 & 36983 & 8324 \\
\hline Electricity, gas \& water supply [85-87] & 627 & 1028 & 12487 & 26717 & 358 & 2633 & 1159 & 2147 & 1502 & 2626 & 811 \\
\hline Construction [88] & 280 & 767 & 1062 & 720 & 63570 & 1928 & 2332 & 15293 & 5313 & 1254 & 755 \\
\hline Distribution \& hotels [89-92] & 772 & 165 & 1537 & 322 & 2040 & 8050 & 3831 & 7284 & 2029 & 2937 & 838 \\
\hline Transport \& communication [93-99] & 554 & 1425 & 15657 & 475 & 1616 & 35310 & 42631 & 29243 & 5731 & 6692 & 3109 \\
\hline Finance \& business services $[100-114]$ & 2827 & 3986 & 38205 & 4798 & 27691 & 66029 & 32720 & 193292 & 25082 & 27671 & 21538 \\
\hline Public administration \& defence [115] & 12 & 31 & 648 & 58 & 487 & 283 & 2209 & 8536 & 318 & 105 & 91 \\
\hline Education, health \& social work [116-118] & 217 & 59 & 1417 & 237 & 222 & 1166 & 1360 & 6448 & 5368 & 39424 & 1164 \\
\hline Other services [119-123] & 328 & 166 & 3749 & 221 & 205 & 2230 & 2112 & 5362 & 3766 & 4386 & 19106 \\
\hline Total consumption & 16716 & 15920 & 316094 & 67469 & 133247 & 172512 & 113821 & 284632 & 73865 & 122401 & 55844 \\
\hline Taxes less subsidies on production & -2864 & 688 & 2499 & 1268 & 945 & 9288 & 873 & 2644 & - & 402 & 1041 \\
\hline Compensation of employees & 4488 & 3914 & 107901 & 6249 & 41994 & 119477 & 62387 & 179913 & 55509 & 145204 & 42155 \\
\hline Gross operating surplus & 8091 & 33116 & 39898 & 13825 & 37817 & 54821 & 28087 & 237423 & 9581 & 24662 & 22367 \\
\hline Gross value added at basic prices & 9715 & 37718 & 150298 & 21342 & 80756 & 183586 & 91347 & 419980 & 65090 & 170268 & 65563 \\
\hline Output at basic prices & 26431 & 53638 & 466392 & 88811 & 214003 & 356098 & 205168 & 704612 & 138955 & 292669 & 121407 \\
\hline
\end{tabular}




\section{Gross value added at basic prices}

$\mathrm{f}$ billion

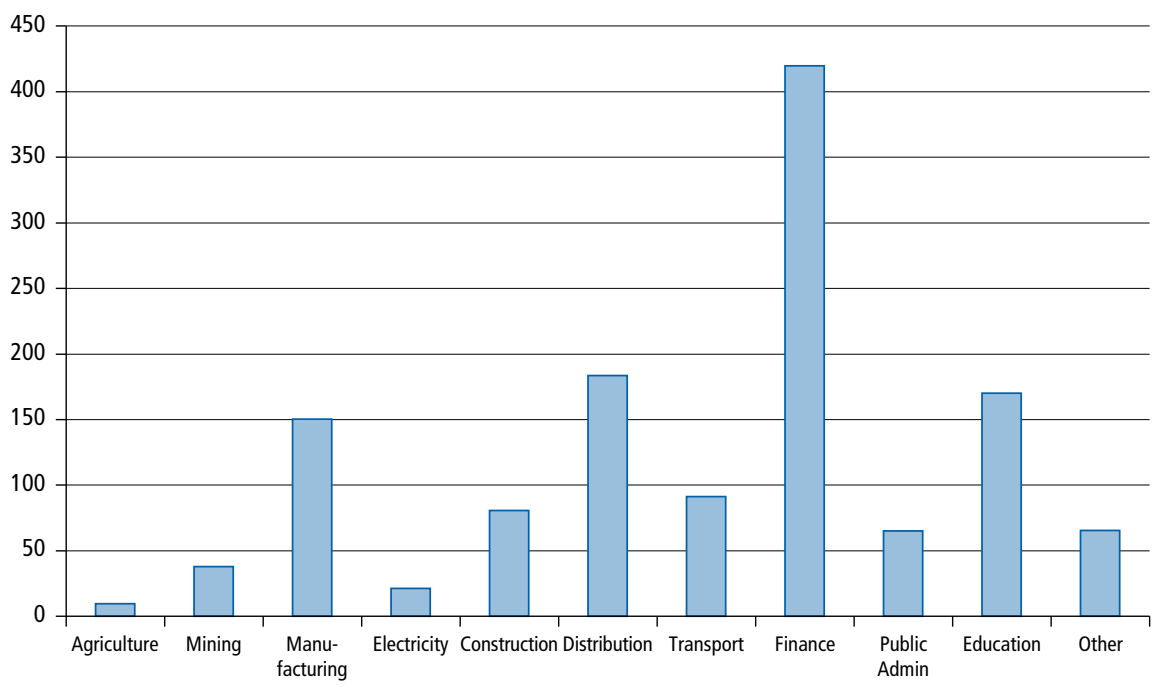

\section{Components of final demand}

Per cent

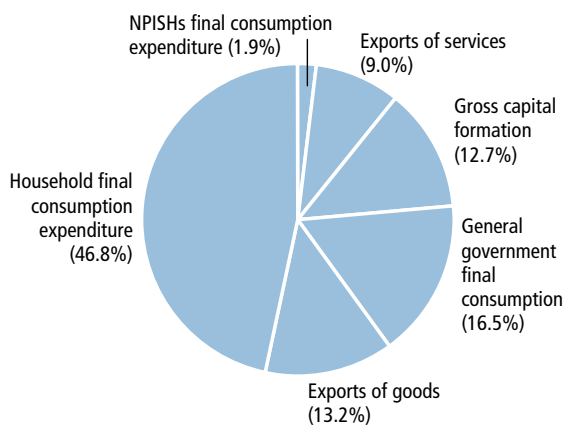

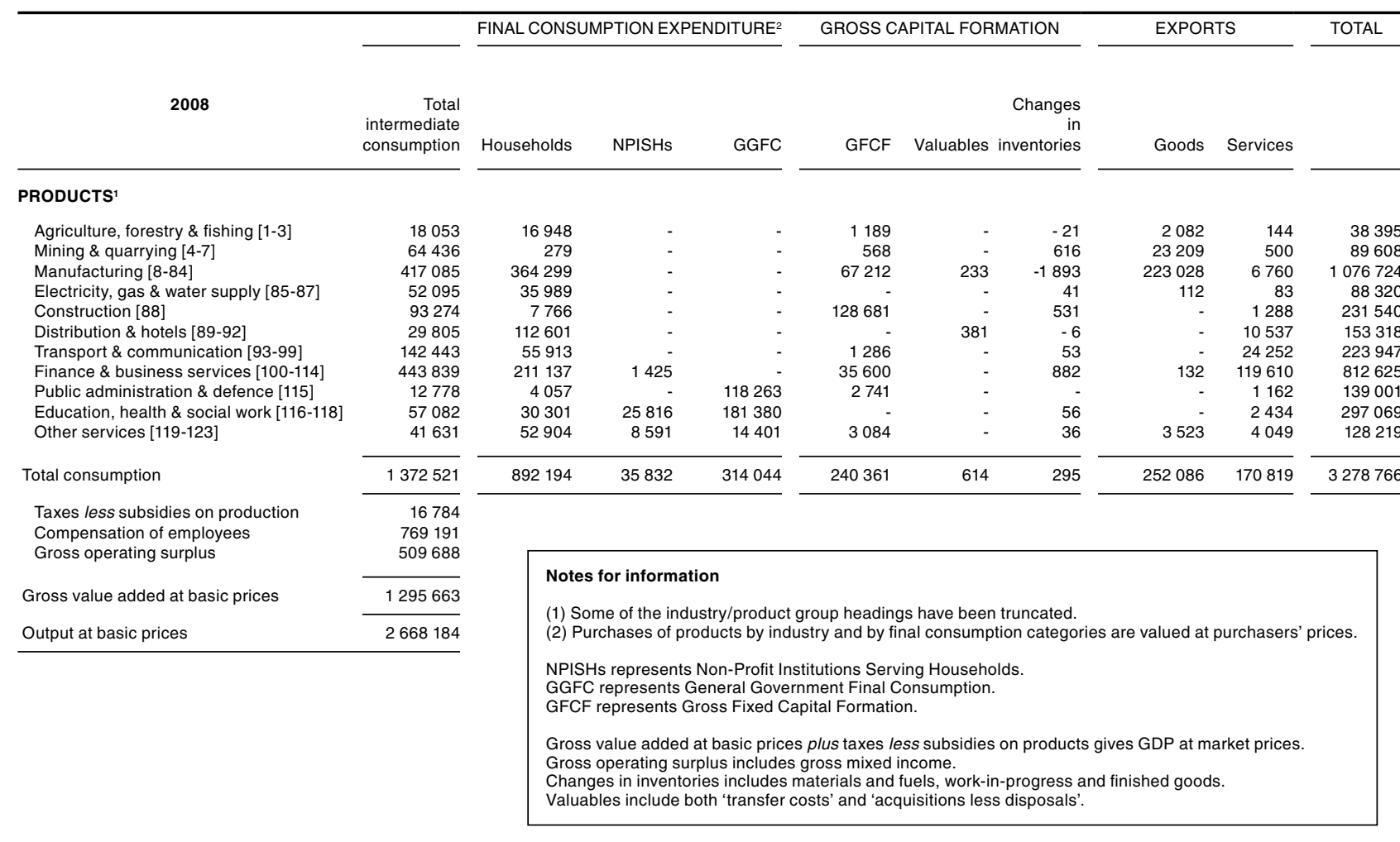

£ million 


\section{Output and capital formation: by industry ${ }^{1,2}$ \\ 2. 2 Gross value added at current basic prices}

f million

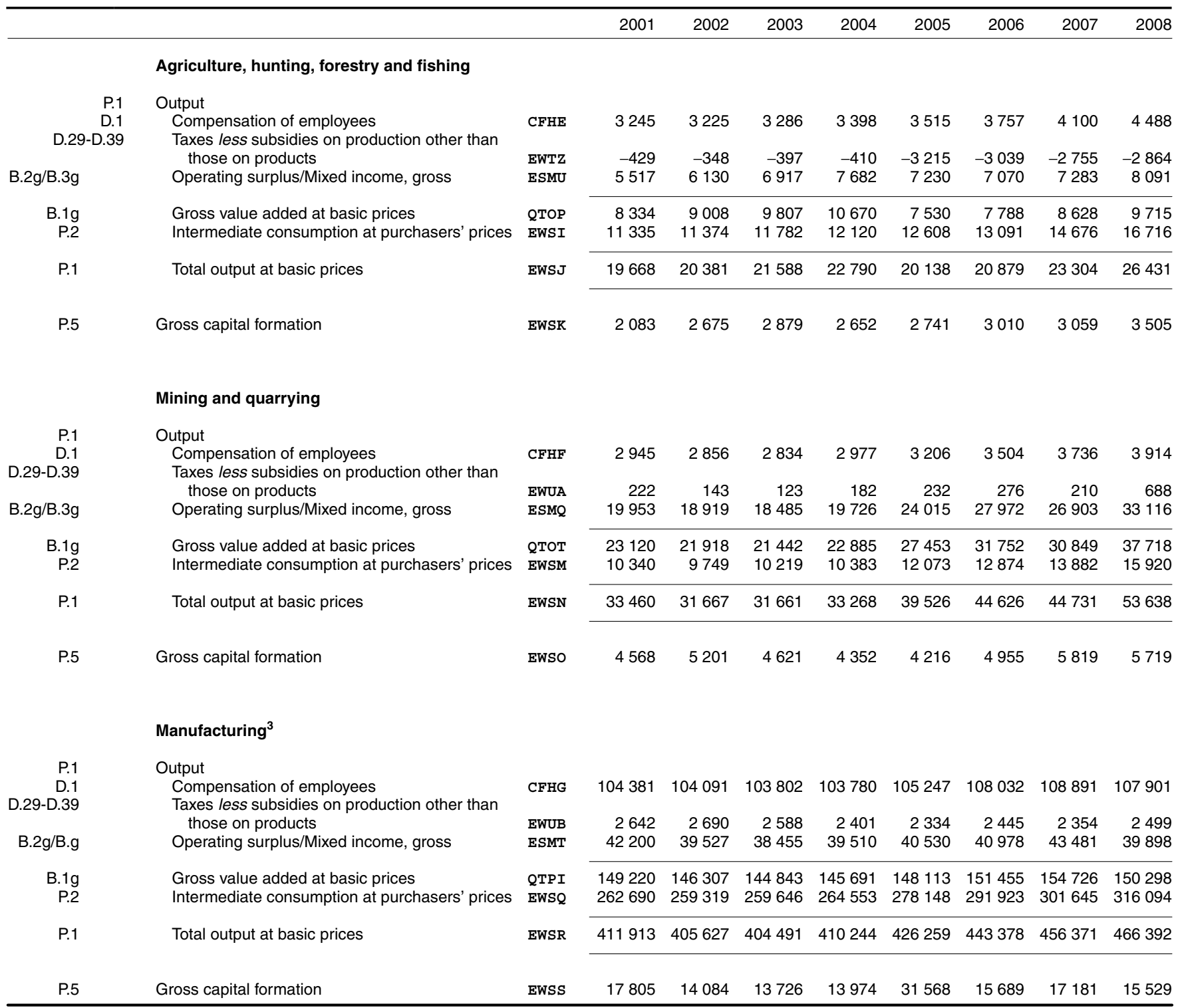

1 The contribution of each industry to the gross domestic product before pro-

viding for consumption of fixed capital. The industrial composition in this

table is consistent with the Input-Output Supply and Use Tables in Table

2.1, which show data from 2006-2008

2 Components may not sum to totals due to rounding.

3 Further detail is given in Table 2.3. 


\section{Output and capital formation: by industry ${ }^{1,2}$ Gross value added at current basic prices}

continued $f$ million

\begin{tabular}{|c|c|c|c|c|c|c|c|c|c|c|}
\hline & & & 2001 & 2002 & 2003 & 2004 & 2005 & 2006 & 2007 & 2008 \\
\hline & \multicolumn{10}{|l|}{ Electricity, gas and water supply } \\
\hline P.1 & Output & & & & & & & & & \\
\hline D.1 & Compensation of employees & CFHI & 4497 & 4606 & 4762 & 4712 & 4857 & 5146 & 6032 & 6249 \\
\hline D.29-D.39 & $\begin{array}{l}\text { Taxes less subsidies on production other than } \\
\text { those on products }\end{array}$ & FWTIC & 1277 & 1214 & 1233 & 1168 & 1117 & & 1132 & 1268 \\
\hline B.2g/B.3g & Operating surplus/Mixed income, gross & ESMV & 9886 & 10232 & 10410 & $\begin{array}{rl}1 & 168 \\
10226\end{array}$ & 10711 & $\begin{array}{r}1149 \\
13984\end{array}$ & $\begin{array}{r}1132 \\
14720\end{array}$ & 13825 \\
\hline B.1g & Gross value added at basic prices & QTPJ & 15660 & 16052 & 16405 & 16106 & 16685 & 20279 & 21884 & 21342 \\
\hline P.2 & Intermediate consumption at purchasers' prices & EWSU & 32872 & 31940 & 32676 & 35716 & 43123 & 51348 & 54494 & 67469 \\
\hline P.1 & Total output at basic prices & EWSV & 48532 & 47992 & 49081 & 51822 & 59808 & 71627 & 76378 & 88811 \\
\hline \multirow[t]{2}{*}{ P.5 } & Gross capital formation & EWSW & 5943 & 5204 & 5288 & 2346 & 4000 & 5164 & 7040 & 8337 \\
\hline & Construction & & & & & & & & & \\
\hline P.1 & Output & & & & & & & & & \\
\hline D.1 & Compensation of employees & CFHU & 25714 & 27472 & 29302 & 30924 & 33320 & 36392 & 41125 & 41994 \\
\hline D.29-D.39 & $\begin{array}{l}\text { Taxes less subsidies on production other than } \\
\text { those on products }\end{array}$ & EWUD & 512 & 533 & 565 & 633 & 610 & 668 & 792 & 945 \\
\hline B. $2 g / B .3 g$ & Operating surplus/Mixed income, gross & ESMW & 24300 & 26679 & 29655 & 34472 & 35938 & 37559 & 38758 & 37817 \\
\hline B. $1 \mathrm{~g}$ & Gross value added at basic prices & QTPL & 50526 & 54684 & 59522 & 66029 & 69868 & 74619 & 80675 & 80756 \\
\hline P.2 & Intermediate consumption at purchasers' prices & EWSY & 82918 & 90607 & 98422 & 105602 & 110094 & 116933 & 130457 & 133247 \\
\hline P.1 & Total output at basic prices & EWSZ & 133444 & 145291 & 157944 & 171631 & 179962 & 191552 & 211132 & 214003 \\
\hline \multirow[t]{2}{*}{ P.5 } & Gross capital formation & EWTA & 3803 & 3783 & 4234 & 3892 & 4238 & 2252 & 7576 & 3154 \\
\hline & Distribution, hotels and catering & & & & & & & & & \\
\hline P.1 & Output & & & & & & & & & \\
\hline D.1 & Compensation of employees & CFIK & 88158 & 91802 & 96364 & 101313 & 104180 & 109702 & 115171 & 119477 \\
\hline D.29-D.39 & $\begin{array}{l}\text { Taxes less subsidies on production other than } \\
\text { those on products }\end{array}$ & EWUE & 7250 & 7330 & 7515 & 7602 & 8300 & 8.423 & 8723 & 9288 \\
\hline B.2g/B.3g & Operating surplus/Mixed income, gross & ESMX & 41769 & 43284 & 46761 & 50322 & 50232 & 51835 & 53803 & 54821 \\
\hline B. $1 \mathrm{~g}$ & Gross value added at basic prices & EWTB & 137177 & 142416 & 150640 & 159237 & 162712 & 169960 & 177697 & 183586 \\
\hline P.2 & Intermediate consumption at purchasers' prices & EWTC & 133316 & 137498 & 143798 & 148250 & 153119 & 158562 & 166696 & 172512 \\
\hline P.1 & Total output at basic prices & EWTD & 270493 & 279914 & 294438 & 307487 & 315831 & 328522 & 344393 & 356098 \\
\hline P.5 & Gross capital formation & EWTE & 20542 & 20628 & 19449 & 26622 & 23559 & 25769 & 29520 & 24151 \\
\hline
\end{tabular}

See footnotes on first page of this table. 


\section{Output and capital formation: by industry ${ }^{1,2}$ 2. 2 Gross value added at current basic prices}

continued f million

\begin{tabular}{|c|c|c|c|c|c|c|c|c|c|c|}
\hline & & & 2001 & 2002 & 2003 & 2004 & 2005 & 2006 & 2007 & 2008 \\
\hline & \multicolumn{10}{|l|}{ Transport, storage and communication } \\
\hline P.1 & Output & & & & & & & & & \\
\hline $\begin{array}{r}\text { D.1 } \\
\text { D.29-D.39 }\end{array}$ & $\begin{array}{l}\text { Compensation of employees } \\
\text { Taxes less subsidies on production other than }\end{array}$ & CFIM & 48434 & 50638 & 51796 & 53187 & 54517 & 55362 & 58830 & 62387 \\
\hline B.2g/B.3g & $\begin{array}{l}\text { those on products } \\
\text { Operating surplus/Mixed income, gross }\end{array}$ & $\begin{array}{l}\text { EWUF } \\
\text { ESMY }\end{array}$ & $\begin{array}{r}1195 \\
20873\end{array}$ & $\begin{array}{r}1495 \\
20931\end{array}$ & $\begin{array}{r}1341 \\
23450\end{array}$ & $\begin{array}{r}1130 \\
24703\end{array}$ & $\begin{array}{r}1237 \\
25135\end{array}$ & $\begin{array}{r}1179 \\
27114\end{array}$ & $\begin{array}{r}831 \\
28619\end{array}$ & $\begin{array}{r}873 \\
28087\end{array}$ \\
\hline $\begin{array}{r}\text { B.1g } \\
\text { P.2 }\end{array}$ & $\begin{array}{l}\text { Gross value added at basic prices } \\
\text { Intermediate consumption at purchasers' prices }\end{array}$ & $\begin{array}{l}\text { QTPQ } \\
\text { EWTG }\end{array}$ & $\begin{array}{l}70501 \\
79429\end{array}$ & $\begin{array}{l}73063 \\
81370\end{array}$ & $\begin{array}{l}76588 \\
87198\end{array}$ & $\begin{array}{l}79020 \\
92702\end{array}$ & $\begin{array}{l}80887 \\
99517\end{array}$ & $\begin{array}{r}83655 \\
103946\end{array}$ & $\begin{array}{r}88280 \\
108696\end{array}$ & $\begin{array}{r}91347 \\
113821\end{array}$ \\
\hline P.1 & Total output at basic prices & EWTH & 149931 & 154434 & 163785 & 171722 & 180406 & 187601 & 196976 & 205168 \\
\hline \multirow[t]{2}{*}{ P.5 } & Gross capital formation & EWTI & 26016 & 24937 & 23633 & 23974 & 18914 & 22109 & 22064 & 24459 \\
\hline & Business services and finance & & & & & & & & & \\
\hline P.1 & Output & & & & & & & & & \\
\hline $\begin{array}{r}\text { D.1 } \\
\text { D.29-D.39 }\end{array}$ & $\begin{array}{l}\text { Compensation of employees } \\
\text { Taxes less subsidies on production other than }\end{array}$ & CFIP & 123101 & 127913 & 136240 & 146025 & 154923 & 166422 & 181073 & 179913 \\
\hline B.2g/B.3g & $\begin{array}{l}\text { those on products } \\
\text { Operating surplus/Mixed income, gross }\end{array}$ & $\begin{array}{l}\text { EWUG } \\
\text { ESMZ }\end{array}$ & $\begin{array}{r}2690 \\
126451\end{array}$ & $\begin{array}{r}2495 \\
147807\end{array}$ & $\begin{array}{r}2411 \\
165083\end{array}$ & $\begin{array}{r}2702 \\
175067\end{array}$ & $\begin{array}{r}1897 \\
182849\end{array}$ & $\begin{array}{r}1936 \\
198557\end{array}$ & $\begin{array}{r}2366 \\
217247\end{array}$ & $\begin{array}{r}2644 \\
237423\end{array}$ \\
\hline $\begin{array}{r}\text { B. } 1 \mathrm{~g} \\
\text { P.2 }\end{array}$ & $\begin{array}{l}\text { Gross value added at basic prices } \\
\text { Intermediate consumption at purchasers' prices }\end{array}$ & $\begin{array}{l}\text { EWTJ } \\
\text { EWTK }\end{array}$ & $\begin{array}{l}252242 \\
194053\end{array}$ & $\begin{array}{l}278215 \\
198288\end{array}$ & $\begin{array}{l}303734 \\
205417\end{array}$ & $\begin{array}{l}323794 \\
208010\end{array}$ & $\begin{array}{l}339669 \\
224936\end{array}$ & $\begin{array}{l}366915 \\
242747\end{array}$ & $\begin{array}{l}400686 \\
258277\end{array}$ & $\begin{array}{l}419980 \\
284632\end{array}$ \\
\hline P.1 & Total output at basic prices & EWTL & 446295 & 476503 & 509151 & 531804 & 564605 & 609662 & 658963 & 704612 \\
\hline \multirow[t]{2}{*}{ P.5 } & Gross capital formation & EWTM & 32908 & 32958 & 34249 & 22660 & 29030 & 30390 & 32167 & 33089 \\
\hline & Public administration and defence & & & & & & & & & \\
\hline P.1 & Output & & & & & & & & & \\
\hline $\begin{array}{r}\text { D.1 } \\
\text { D.29-D.39 }\end{array}$ & $\begin{array}{l}\text { Compensation of employees } \\
\text { Taxes less subsidies on production other than }\end{array}$ & CFIV & 38450 & 40608 & 44035 & 47713 & 51853 & 53573 & 54245 & 55509 \\
\hline & those on products & EWUH & - & - & - & - & - & - & - & - \\
\hline B.2g & Operating surplus, gross & EWUW & 6575 & 6920 & 7267 & 7771 & 8213 & 8651 & 9063 & 9581 \\
\hline B.1g & Gross value added at basic prices & QTPV & 45025 & 47528 & 51302 & 55485 & 60066 & 62224 & 63308 & 65090 \\
\hline P.2 & Intermediate consumption at purchasers' prices & EWTO & 43236 & 48394 & 52942 & 59722 & 63511 & 67577 & 69018 & 73865 \\
\hline P.1 & Total output at basic prices & EWTP & 88261 & 95922 & 104244 & 115206 & 123577 & 129801 & 132326 & 138955 \\
\hline P.5 & Gross capital formation & EWTQ & 6987 & 8490 & 11141 & 12214 & 572 & 15672 & 18027 & 23560 \\
\hline
\end{tabular}

See footnotes on first page of this table. 


\section{Output and capital formation: by industry ${ }^{1,2}$ Gross value added at current basic prices}

continued f million

\begin{tabular}{|c|c|c|c|c|c|c|c|c|c|c|}
\hline & & & 2001 & 2002 & 2003 & 2004 & 2005 & 2006 & 2007 & 2008 \\
\hline & \multicolumn{10}{|l|}{ Education, health and social work } \\
\hline P.1 & Output & & & & & & & & & \\
\hline D. 1 & Compensation of employees & CFIW & 96724 & 103787 & 112124 & 118425 & 125731 & 133217 & 138945 & 145204 \\
\hline D.29-D.39 & Taxes less subsidies on production other & & & & & & & & & \\
\hline & than those on products & EWUI & 130 & 190 & 152 & 245 & 282 & 343 & 340 & 402 \\
\hline B. $2 \mathrm{~g} / \mathrm{B} .3 \mathrm{~g}$ & Operating surplus/Mixed income, gross & EWSF & 14370 & 15614 & 16645 & 18418 & 19691 & 21331 & 22862 & 24662 \\
\hline $\begin{array}{r}\text { B. } 1 \mathrm{~g} \\
\text { P. } 2\end{array}$ & purchasers' prices & EWTS & 66240 & 73261 & 80745 & 88409 & 96448 & 105248 & 113143 & 122401 \\
\hline P.1 & Total output at basic prices & EWTT & 177464 & 192852 & 209666 & 225497 & 242152 & 260139 & 275290 & 292669 \\
\hline P.5 & Gross capital formation & EWTU & 8119 & 8630 & 9447 & 13599 & 11063 & 13771 & 14226 & 17736 \\
\hline
\end{tabular}

\section{Other services}

$\begin{aligned} & \text { P.1 } \text { Output } \\ & \text { D.1 } \text { Compensation of employees } \\ & \text { Taxes less subsidies on production other } \\ & \text { than those on products } \\ & \text { Operating surplus/Mixed income, gross } \\ & \text { B.2g/B.3g } \begin{array}{l}\text { Gross value added at basic prices } \\ \text { Intermediate consumption at } \\ \text { purchasers' prices }\end{array} \\ & \text { P. } 2 \text { Total output at basic prices } \\ & \text { P.1 } \text { Gross capital formation }\end{aligned}$

\begin{tabular}{lrrrrrrrr} 
CFIX & 28545 & 30398 & 32348 & 33897 & 36129 & 37901 & 40066 & 42155 \\
EWUJ & 832 & 898 & 960 & 1046 & 1055 & 1139 & 1097 & 1041 \\
EWSG & 15184 & 17016 & 18496 & 20004 & 20777 & 21126 & 21661 & 22367 \\
\cline { 2 - 8 } QTPY & 44559 & 48309 & 51801 & 54947 & 57962 & 60166 & 62824 & 65563 \\
EWTW & 36988 & 40640 & 42322 & 44475 & 47536 & 50164 & 51625 & 55844 \\
\cline { 2 - 8 } EWTX & 81549 & 88952 & 94126 & 99422 & 105497 & 110330 & 114449 & 121407 \\
\cline { 2 - 8 } EWTY & 9244 & 9735 & 11000 & 14161 & 16316 & 15630 & 16366 & 17315
\end{tabular}

Not allocated to industries

\section{All industries}

\begin{tabular}{|c|c|}
\hline P.1 & Output \\
\hline D.1 & Compensation of employees \\
\hline D.29-D.39 & $\begin{array}{l}\text { Taxes less subsidies on production other } \\
\text { than those on products }\end{array}$ \\
\hline B.2g & Operating surplus, gross \\
\hline B. $3 g$ & Mixed income, gross \\
\hline di & $\begin{array}{l}\text { Statistical discrepancy between income } \\
\text { and GDP }\end{array}$ \\
\hline B. $1 \mathrm{~g}$ & Gross value added at basic prices \\
\hline P.2 & $\begin{array}{l}\text { Intermediate consumption at } \\
\text { purchasers' prices }\end{array}$ \\
\hline P.1 & Total output at basic prices \\
\hline P.5 & Gross capital formation \\
\hline P.51 & Gross fixed capital formation \\
\hline P.52 & Changes in inventories \\
\hline P.53 & Acquisitions less disposals of valuables \\
\hline P.5 & Total gross capital formation \\
\hline
\end{tabular}

\begin{tabular}{|c|c|c|c|c|c|c|c|c|}
\hline HAEA & 564194 & 587396 & 616893 & 646351 & 677478 & 713008 & 752214 & 769191 \\
\hline QZPC & 16321 & 16640 & 16491 & 16699 & 13849 & 14519 & 15090 & 16784 \\
\hline $\mathrm{ABNF}$ & 265797 & 288091 & 313300 & 333619 & 346260 & 375013 & 402440 & 424804 \\
\hline QWLT & 61282 & 64967 & 68324 & 74282 & 79061 & 81164 & 81960 & 84884 \\
\hline RVFC & - & - & - & - & - & - & - & - \\
\hline ABML & 907594 & 957094 & 1015008 & 1070951 & 1116648 & 1183704 & 1251704 & 1295663 \\
\hline NQAJ & 953417 & 982440 & 1025167 & 1069942 & 1141113 & 1214413 & 1282609 & 1372521 \\
\hline NQAF & 1861011 & 1939534 & 2040175 & 2140893 & 2257761 & 2398117 & 2534313 & 2668184 \\
\hline NPQX & 171782 & 180551 & 186700 & 200415 & 209758 & 227234 & 249517 & 240361 \\
\hline ABMP & 6189 & 2909 & 3983 & 4886 & 4472 & 5212 & 5815 & 295 \\
\hline NPJO & 396 & 214 & -37 & -37 & -376 & 285 & 374 & 614 \\
\hline JQFM & 178367 & 183674 & 190646 & 205264 & 213854 & 232731 & 255706 & 241270 \\
\hline
\end{tabular}

See footnotes on first page of this table. 


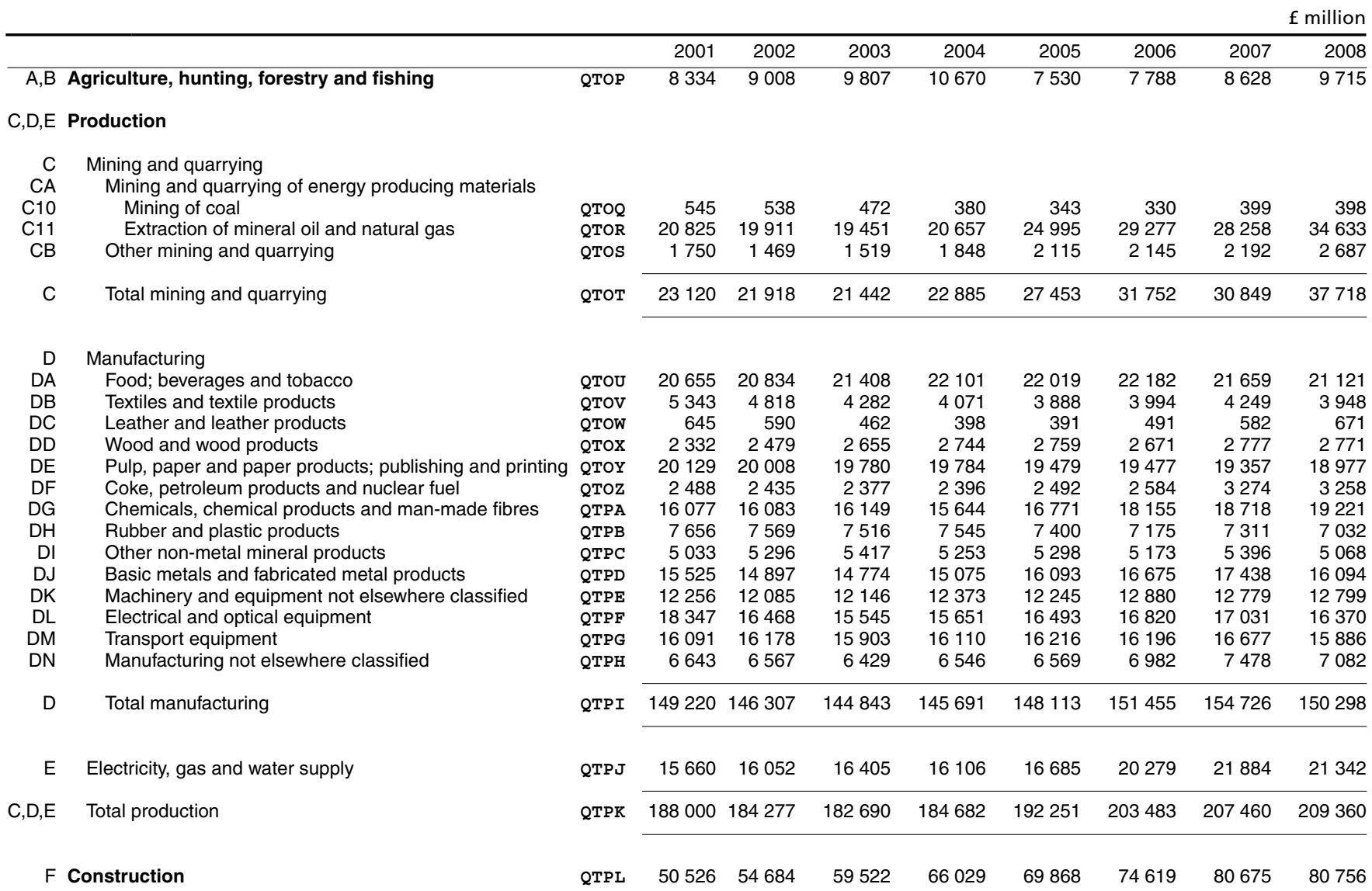

\section{G-Q Service industries}

G Wholesale and retail trade (including motor trade); repair of motor vehicles, personal and household goods QTPM $\quad 110250113776 \quad 120520 \quad 127366 \quad 129811 \quad 135366 \quad 141735 \quad 147159$

H Hotels and restaurants

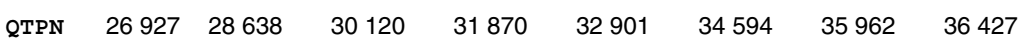

I Transport, storage and communication Transport and storage Communication

I Total $\begin{array}{lllllllll}\text { QTPO } & 43184 & 44501 & 47022 & 48703 & 50203 & 53090 & 55866 & 59066 \\ \text { QTPP } & 27317 & 28562 & 29566 & 30317 & 30684 & 30565 & 32414 & 32281\end{array}$

\begin{tabular}{lllllllll}
\hline QTPQ & 70501 & 73063 & 76588 & 79020 & 80887 & 83655 & 88280 & 91347
\end{tabular}

$\mathrm{J}$ Financial intermediation $\begin{array}{lllllllll}\text { QTPR } & 48202 & 63368 & 71530 & 75117 & 79554 & 90806 & 103730 & 116801\end{array}$

K Real estate, renting and business activities Letting of dwellings including imputed rent of owner occupiers Other real estate, renting and business activities

K Total $\begin{array}{lrrrrrrrr}\text { QTPT } & 142689 & 150599 & 162909 & 174427 & 183299 & 196019 & 208749 & 215669\end{array}$

\begin{tabular}{lllllllll}
\cline { 2 - 2 } & 204041 & 214848 & 232207 & 248676 & 260116 & 276109 & 296956 & 303179
\end{tabular}

L Public administration and defence (PAD)

$\begin{array}{lllllllll}\text { QTPV } & 45025 & 47528 & 51302 & 55485 & 60066 & 62224 & 63308 & 65090\end{array}$

M Education

$\mathrm{N} \quad$ Health and social work

$\begin{array}{lllllllll}\text { QTPW } & 51675 & 55099 & 58328 & 61934 & 65739 & 68926 & 72766 & 76493\end{array}$

$\begin{array}{lllllllll}\text { QTPX } & 59549 & 64493 & 70592 & 75154 & 79965 & 85965 & 89381 & 93775\end{array}$

$\mathrm{O}, \mathrm{P}, \mathrm{Q}$ Other social and personal services, private households with employees and extra-territorial organisations

G-Q Total service industries

\begin{tabular}{lllllllll} 
QTPY & 44559 & 48309 & 51801 & 54947 & 57962 & 60166 & 62824 & 65563 \\
\hline
\end{tabular}

$\begin{array}{lllllllll}\text { QTPZ } & 660729 & 709122 & 762988 & 809569 & 847001 & 897814 & 954941 & 995832\end{array}$

1 Components may not sum to totals as a result of rounding.

2 Because of differences in the annual and monthly production inquiries, esti-

mates of current price output and gross value added by industry derived

from the current price Input-Output Supply and Use Tables are not consis-

tent with the equivalent measures of chained volume measures growth giv-

en in 2.4. These differences do not affect GDP totals. 


\section{Gross value added at basic prices: by industry ${ }^{1,2,3}$ \\ Chained volume indices}

Indices $2006=100$

\begin{tabular}{lllll}
\hline & Weight per 1000' & & & \\
& $2001 \quad 2002 \quad 2003 \quad 2004 \quad 2005 \quad 2006 \quad 2007 \quad 2008 \quad 2009$ \\
\hline
\end{tabular}

$A, B$ Agriculture, hunting, forestry and fishing

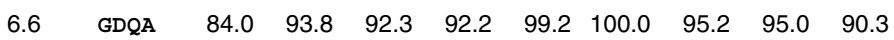

\section{C,D,E Production}

C Mining and quarrying

$\mathrm{CA} \quad$ Mining and quarrying of energy producing materials

C10 Mining of coal

C11 Extraction of mineral oil and natural gas

$\mathrm{CB}$ Other mining and quarrying

C Total mining and quarrying

\begin{tabular}{rrrrrrrrrrr}
0.3 & CKZP & 172.8 & 162.1 & 153.1 & 133.3 & 102.9 & 100.0 & 86.0 & 92.2 & 89.1 \\
24.7 & CKZO & 143.0 & 141.3 & 133.5 & 122.7 & 110.1 & 100.0 & 97.5 & 92.6 & 85.8 \\
1.8 & CKZQ & 68.2 & 82.7 & 83.7 & 85.8 & 94.2 & 100.0 & 106.8 & 82.1 & 48.9 \\
\hline \multirow{2}{*}{26.8} & \multirow{2}{*}{ CKYX } & 135.2 & 135.7 & 128.9 & 119.1 & 108.6 & 100.0 & 98.1 & 91.9 & 83.3 \\
\hline
\end{tabular}

D Manufacturing

DA Food; beverages and tobacco

Textiles and textile products

Leather and leather products

Wood and wood products

Pulp, paper and paper products; publishing and printing

Coke, petroleum products and nuclear fuel

Chemicals, chemical products and man-made fibres

Rubber and plastic products

Other non-metallic mineral products

Basic metals and fabricated metal products

Machinery and equipment not elsewhere classified

Electrical and optical equipment

Transport equipment

Manufacturing not elsewhere classified

D Total manufacturing

E Electricity, gas and water supply

C,D,E Total production

F Construction

\begin{tabular}{rlrrrrrrrrr}
18.7 & CKZA & 96.1 & 98.8 & 97.1 & 98.7 & 100.7 & 100.0 & 100.0 & 98.3 & 96.4 \\
3.4 & CKZB & 118.1 & 111.3 & 111.7 & 102.1 & 100.6 & 100.0 & 98.0 & 98.2 & 89.6 \\
0.4 & CKZC & 183.0 & 166.3 & 142.7 & 104.9 & 95.7 & 100.0 & 102.9 & 97.3 & 91.4 \\
2.3 & CKZD & 99.0 & 99.8 & 101.2 & 106.5 & 102.4 & 100.0 & 104.1 & 97.2 & 81.2 \\
16.5 & CKZE & 107.5 & 107.7 & 105.8 & 104.5 & 100.5 & 100.0 & 100.3 & 98.0 & 89.6 \\
2.2 & CKZF & 102.0 & 104.2 & 95.4 & 107.2 & 105.9 & 100.0 & 102.4 & 104.7 & 99.3 \\
15.3 & CKZG & 89.5 & 90.2 & 91.0 & 94.5 & 97.0 & 100.0 & 99.1 & 99.0 & 94.2 \\
6.1 & CKZH & 101.9 & 98.0 & 98.8 & 97.1 & 96.0 & 100.0 & 99.1 & 94.1 & 81.5 \\
4.4 & CKZI & 88.8 & 87.8 & 91.7 & 97.2 & 97.3 & 100.0 & 100.0 & 94.5 & 81.2 \\
14.1 & CKZJ & 95.3 & 96.4 & 95.1 & 97.7 & 98.5 & 100.0 & 100.7 & 96.7 & 78.2 \\
10.9 & CKZK & 89.7 & 84.9 & 85.9 & 91.0 & 93.9 & 100.0 & 103.8 & 102.1 & 82.0 \\
14.2 & CKZL & 122.4 & 106.3 & 102.4 & 104.3 & 100.3 & 100.0 & 98.7 & 92.7 & 83.2 \\
13.7 & CKZM & 89.5 & 86.7 & 90.9 & 96.0 & 95.6 & 100.0 & 100.9 & 98.4 & 84.2 \\
5.9 & CKZN & 97.3 & 98.4 & 98.3 & 97.6 & 99.0 & 100.0 & 103.7 & 97.8 & 89.3 \\
\hline 128.0 & \multirow{2}{*}{ CKYY } & 98.9 & 96.8 & 96.5 & 98.6 & 98.5 & 100.0 & 100.5 & 97.6 & 87.3 \\
\hline 17.1 & CKYZ & 97.6 & 98.1 & 99.8 & 100.9 & 100.5 & 100.0 & 100.2 & 100.3 & 91.8 \\
\hline 171.9 & \multirow{2}{*}{ CKYW } & 102.5 & 100.8 & 100.2 & 101.2 & 100.0 & 100.0 & 100.1 & 97.0 & 87.1 \\
\hline & & & & & & & & & & \\
63.0 & \multirow{2}{*}{ GDQB } & 86.8 & 90.2 & 94.8 & 98.0 & 99.0 & 100.0 & 102.7 & 101.9 & 90.7
\end{tabular}

\section{G-Q Service industries}

G Wholesale and retail trade (including motor trade);

repair of motor vehicles, personal and household goods

$\begin{array}{lllllllllll}114.4 & \text { GDQC } & 82.4 & 87.0 & 90.0 & 95.7 & 97.0 & 100.0 & 102.6 & 99.9 & 96.1\end{array}$

H Hotels and restaurants

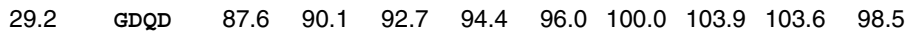

I Transport, storage and communication

Transport and storage

Communication

I Total

$\mathrm{J}$ Financial intermediation

$\begin{array}{lllllllllll}44.9 & \text { GDQF } & 89.4 & 90.7 & 89.5 & 94.3 & 97.4 & 100.0 & 103.1 & 103.6 & 94.5\end{array}$

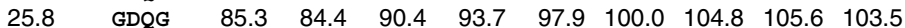

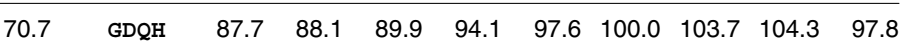

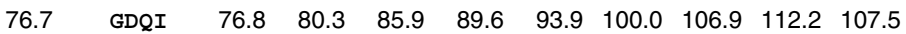

K Real estate, renting and business activities

Letting of dwellings, including imputed rent

of owner occupiers

Other real estate, renting and business activities

K Total

L Public administration and defence (PAD)

M Education

$\mathrm{N} \quad$ Health and social work

P,Q Other social and personal services,

private households with employees

and extra-territorial organisations

G-Q Total service industries

\begin{tabular}{rlrllllllll}
67.7 & GDQL & 90.5 & 91.3 & 92.9 & 92.3 & 92.9 & 100.0 & 100.9 & 102.0 & 103.2 \\
165.6 & GDQK & 78.1 & 78.3 & 82.0 & 87.2 & 93.5 & 100.0 & 106.9 & 107.9 & 100.5 \\
\hline 233.3 & GDQM & 81.5 & 81.9 & 85.0 & 88.7 & 93.3 & 100.0 & 105.2 & 106.2 & 101.2 \\
\hline \multirow{2}{*}{52.6} & \multirow{2}{*}{ GDQO } & 90.3 & 92.8 & 97.6 & 98.7 & 99.5 & 100.0 & 100.1 & 97.8 & 100.1 \\
58.2 & \multirow{2}{*}{ GDQP } & 97.2 & 99.2 & 100.1 & 99.5 & 100.1 & 100.0 & 99.9 & 100.3 & 101.1 \\
72.6 & \multirow{2}{*}{ GDQQ } & 84.8 & 88.3 & 91.7 & 94.7 & 97.5 & 100.0 & 103.0 & 105.2 & 107.3
\end{tabular}

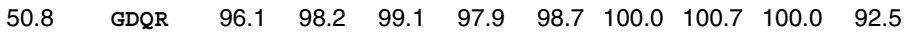

\begin{tabular}{|c|c|c|c|}
\hline 758.5 & 84.9 & 7.0 & 758. \\
\hline
\end{tabular}

B.1g All industries

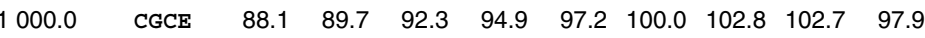

1 The weights shown are in proportion to total gross value added (GVA) in 3 See footnote 2 to Table 2.3 .

2006 and are used to combine the industry output indices to calculate the

totals. For 2005 and earlier, totals are calculated using the equivalent

weights for the previous year (e.g. totals for 2005 use 2004 weights).

Weights may not sum to totals due to rounding

2 As GVA is expressed in index number form, it is inappropriate to show as a

statistical adjustment any divergence from the other measures of GDP.

Such an adjustment does, however, exist implicitly. 
Thousands

\begin{tabular}{|c|c|c|c|c|c|c|c|c|c|c|c|}
\hline & & & 2001 & 2002 & 2003 & 2004 & 2005 & 2006 & 2007 & 2008 & 2009 \\
\hline$A, B$ & Agriculture, hunting \& forestry; fishing & & & & & & & & & & \\
\hline ESE & Self-employment jobs & YEKN & 200 & 201 & 200 & 204 & 215 & 212 & 211 & 233 & 282 \\
\hline EEM & Employee jobs \& government supported trainees & YEKO & 261 & 272 & 246 & 257 & 277 & 271 & 274 & 283 & 244 \\
\hline ETO & Workforce jobs & YEKP & 461 & 473 & 447 & 461 & 492 & 483 & 485 & 516 & 525 \\
\hline C-E & Production industries, including energy & & & & & & & & & & \\
\hline ESE & Self-employment jobs & YEKQ & 266 & 279 & 282 & 290 & 261 & 288 & 283 & 279 & 246 \\
\hline EEM & Employee jobs \& government supported trainees & YEKR & 3992 & 3779 & 3571 & 3396 & 3235 & 3125 & 3098 & 2995 & 2767 \\
\hline ETO & Workforce jobs & YEKS & 4257 & 4058 & 3854 & 3686 & 3496 & 3412 & 3381 & 3273 & 3014 \\
\hline $\mathrm{F}$ & Construction & & & & & & & & & & \\
\hline ESE & Self-employment jobs & YEKT & 696 & 738 & 818 & 848 & 879 & 857 & 928 & 909 & 840 \\
\hline ETO & Workforce jobs & YEKV & 1892 & 1908 & 1990 & 2045 & 2110 & 2152 & 2217 & 2187 & 2063 \\
\hline G-I & $\begin{array}{l}\text { Wholesale \& retail trade (including motor trade); } \\
\text { repair of motor vehicles, personal \& household goods; } \\
\text { hotels and restaurants; } \\
\text { transport, storage \& communication }\end{array}$ & & & & & & & & & & \\
\hline ESE & Self-employment jobs & YEKW & 898 & 902 & 914 & 925 & 875 & 887 & 888 & 889 & 904 \\
\hline EEM & Employee jobs \& government supported trainees & YEKX & 7740 & 7819 & 7848 & 7906 & 7966 & 7950 & 7957 & 8016 & 7719 \\
\hline ETO & Workforce jobs & YEKY & 8638 & 8721 & 8762 & 8831 & 8841 & 8837 & 8845 & 8905 & 8622 \\
\hline $\mathrm{J}-\mathrm{K}$ & $\begin{array}{l}\text { Financial intermediation; } \\
\text { real estate, renting \& business activities }\end{array}$ & & & & & & & & & & \\
\hline $\begin{array}{l}\text { ESE } \\
\text { EEM }\end{array}$ & $\begin{array}{l}\text { Self-employment jobs } \\
\text { Employee jobs \& government supported trainees }\end{array}$ & $\begin{array}{l}\text { YEKZ } \\
\text { YELA }\end{array}$ & $\begin{array}{r}724 \\
5022\end{array}$ & $\begin{array}{r}732 \\
5065\end{array}$ & $\begin{array}{r}828 \\
5088\end{array}$ & $\begin{array}{r}815 \\
5191\end{array}$ & $\begin{array}{r}835 \\
5384\end{array}$ & $\begin{array}{r}813 \\
5602\end{array}$ & $\begin{array}{r}859 \\
5783\end{array}$ & $\begin{array}{r}860 \\
5970\end{array}$ & $\begin{array}{r}886 \\
5713\end{array}$ \\
\hline$L-Q$ & $\begin{array}{l}\text { Other service activities } \\
\text { Public administration \& defence, } \\
\text { education, health and social work, } \\
\text { other community, social \& personal services, } \\
\text { private households with employees }\end{array}$ & & & & & & & & & & \\
\hline $\begin{array}{l}\text { ESE } \\
\text { EEM }\end{array}$ & $\begin{array}{l}\text { Self-employment jobs } \\
\text { Employee jobs, HM Forces \& government supported trainees }\end{array}$ & $\begin{array}{l}\text { YEJW } \\
\text { YEJX }\end{array}$ & 830 & $\begin{array}{r}841 \\
8247\end{array}$ & $\begin{array}{r}872 \\
8497\end{array}$ & $\begin{array}{r}899 \\
8704\end{array}$ & $\begin{array}{r}900 \\
8924\end{array}$ & 988 & $\begin{array}{r}985 \\
8973\end{array}$ & $\begin{array}{l}1007 \\
9051\end{array}$ & $\begin{array}{l}1026 \\
9149\end{array}$ \\
\hline ETO & Workforce jobs & YEJY & 8861 & 9089 & 9369 & 9602 & 9823 & 10021 & 9958 & 10058 & 10174 \\
\hline$A-Q$ & All industries & & & & & & & & & & \\
\hline ESE & Self-employment jobs & BCAG & 3615 & 3692 & 3915 & 3981 & 3965 & 4044 & 4154 & 4177 & 4183 \\
\hline EEM & Employee jobs, HM Forces \& government supported trainees & IK6H & 26240 & 26353 & 26422 & 26651 & 27017 & 27276 & 27374 & 27592 & 26814 \\
\hline ETO & Workforce jobs & YEJZ & 29855 & 30046 & 30337 & 30632 & 30982 & 31320 & 31528 & 31769 & 30997 \\
\hline
\end{tabular}

1 Data sources are: Labour Force Survey for self-employment jobs; employer surveys for employee jobs; administrative sources for HM forces and gov-

ernment supported trainees. Figures as at June of each year. 